\title{
Pulsating star research from Antarctica
}

\author{
Merieme Chadid $^{1, \star}$ \\ ${ }^{1}$ Université Nice Sophia-Antipolis, Observatoire Côte d'Azur, UMR 7293, Parc Valrose, F-06108, Nice \\ Cedex 02, France
}

\begin{abstract}
This invited talk discusses the pulsating star research from the heart of Antarctica and the scientific polar challenges in the extreme environment of Antarctica, and how the new polar technology could cope with unresolved stellar pulsation enigmas and evolutionary properties challenges towards an understanding of the mysteries of the Universe.

PAIX, the first robotic photometer Antarctica program, has been successfully launched during the polar night 2007. This ongoing program gives a new insight to cope with unresolved stellar enigmas and stellar oscillation challenges with a great opportunity to benefit from an access to the best astronomical site on Earth, Dome C. PAIX achieves astrophysical measurement time-series of stellar fields, challenging photometry from space. A continuous and an uninterrupted series of multi-color photometric observations has been collected each polar night - 150 days - without regular interruption, Earth's rotation effect. PAIX shows the first light curve from Antarctica and first step for the astronomy in Antarctica giving new insights in remote polar observing runs and robotic instruments towards a new technology.
\end{abstract}

\section{Introduction}

Stellar pulsations and asteroseismogy are currently among the fundamental techniques to improve our understanding of the internal structure of stars and the hydrodynamics of their atmosphere. On the observational side, progress is limited by the data accuracy needed to detect numerous modes of oscillations with small amplitudes and by the discontinuous nature of typical ground-based data strings which often introduce ambiguities in the determination of oscillation frequencies. Space missions such as MOST ([12]), CoRoT ([1]) and Kepler ([2]) enable to overcome both difficulties, and indeed have considerably enhanced the scope of pulsation and asteroseismology methods. However, the outcome of the space missions on the stellar oscillation fields shows large gaps in terms of the flexibility during the observing runs, the choice of targets, the repair of failures and the inexorable high costs. Now the time has come to implement a new way to study the stellar oscillations with long uninterrupted and continuous observations over 150 days from the ground. PAIX - Photometer AntarctIca eXtinction - is a polar program ([6]) made of low cost commercial components, and achieves astrophysical measurement time-series of stellar fields, challenging photometry from space. and even has more advantages than the space telescopes in observing in $U B V R I$ bands photometric colors and then collecting simultaneously multicolor light curves of several targets within the same $12.4 \times 8.3$ arcmin

\footnotetext{
${ }^{\star}$ chadid@unice. fr
} 
field of view. PAIX has been "antarctized" to run under extreme weather conditions with temperatures as low as $-80^{\circ} \mathrm{C}$, and has been robotized, designed and built by PAIXTeam.

In what follows we summarize a brief review of the PAIX polar mission, the concepts and polarcraft. We discuss the technical and financial gaps between space telescopes and PAIX Polar Mission and then we present the first PAIX outcomes on the stellar pulsation fields.

\section{PAIX first robotic Antarctic photometer}

Gaps in the time variability of stellar brightness or radial velocity are a major problem in pulsating and oscillating study. It is mandatory to measure the frequency spectrum with the narrowest bandwidth $\Delta v$ possible, which means that the observing time $T$ must be as long as possible following the relationship $\Delta v=1 / T$. In classical ground observatories, stars are observed only during few consecutive weeks, so a low duty cycle, which is the percentages of time observed over the span of the data set, brings confusions in determining frequencies caused by the day-night interruptions such as one-day aliases appearing around $v=11.6 \mu \mathrm{Hz}$.

\subsection{Site testing}

Several site testing studies from the heart of Antarctica has been done and show that the best polar astronomical site of observation is the Antarctic plateau Dome C (DMS $75^{\text {deg }} 6^{m n} 0^{s} \mathrm{~S}, 123^{\text {deg }} 21^{m n} 0^{s} \mathrm{E}$, Decimal -75.1, 123.35). A comprehensive set of site testing experiments has been deployed on the Dome $\mathrm{C}$ base, dedicated to astronomical characterization of the optical turbulence and atmosphere transparency such as Differential Image Motion Monitor ([14]), Single Star Scidar ([13]), Scintillation Detection and Ranging ([15]), and PAIX ([3]). These deep investigations showed that Dome C benefits from exceptional astronomical conditions within a large wavelength range, from blue remote millimeter. Several factors allow such good astronomical conditions such as the high altitude $3300 \mathrm{~m}$ a.s.l., the extremely low temperature oscillating between -50 and $-83^{\circ}$ Celsius, the low cloudiness and air-glow emission, the extremely low light pollution and the very pure air. We clearly demonstrated that most of the optical turbulence is concentrated within the first $30 \mathrm{~m}$ of the atmosphere ([10]), and the rest of the atmosphere is very quiet with a seeing of about $0.3-0.4 \operatorname{arcsec}$, as early noticed by [11]. Dome $\mathrm{C}$ lies within the best astronomical places in the world.

We show in Figure 1 that the sky is dark when the Sun elevation is less than -8 and not -18 degrees as generally accepted in classical observatories. With such threshold, stars are tracked without any interruption during several months, and the overall observing duration is 135 days. At classical observatories there is no way to achieve continuous and uninterrupted observation over 24 hours.

\subsection{How does polar pulsation research happen}

PAIX, the first Robotic Antarctica Photometer, launched in 2007 at Dome C, is made of low cost commercial components, and achieves astrophysical measurement multi-color time-series of stellar pulsation fields. The PAIX CCD camera is a SBIG ST10-XME connected to a filter wheel, attached to an Astro-Physics AP3600 equatorial mount which supports a $0.4 \mathrm{~m}$ Ritchey-Chretien telescope (Fig. 2). All telescope systems, motors, encoders, main CCD camera and CCD finder are under a thermal stabilization control. Two remote cameras are placed each side of the telescope, as well as a switchable light, in order to check for whole instrument safeness during the dark winter.

A remote command of the whole instrument, Paix Automatic Control Software (PACS, Fig. 4) allows, from any area in the world equipped with an internet link, to control the status and to pilot the 
Night duration vs sun elevation at tropics $(-30)$

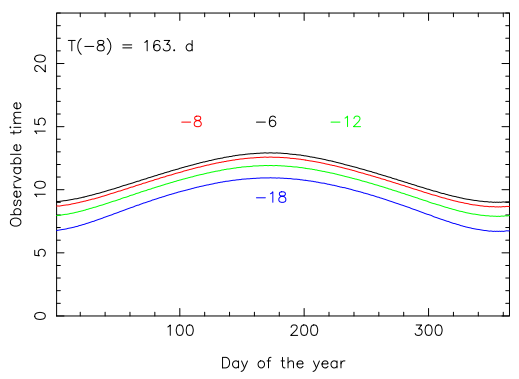

Night duration vs sun elevation at Dome C $(-75)$

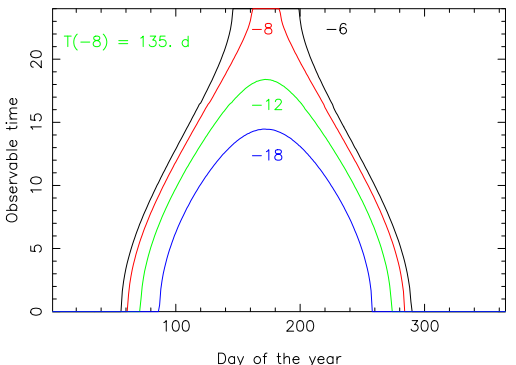

Night duration vs sun elevation at mid-latitude $(-45)$

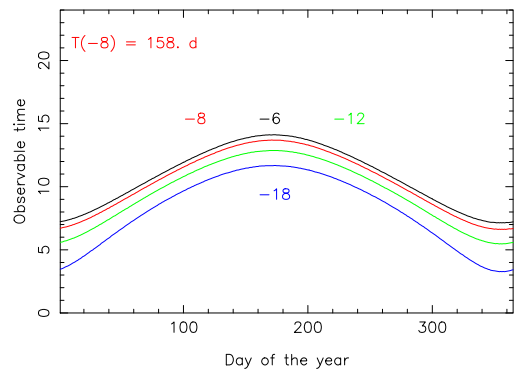

Night duration vs sun elevation at South Pole $(-90)$

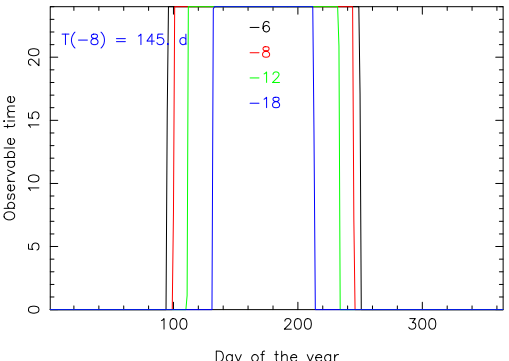

Figure 1. Night duration as a function of the Sun threshold, $-6,-8,-12$ and -18 degrees below horizon, and depending upon the latitude $\lambda=-30,-45,-75$ and -90 degrees. Of course, this night duration is equal to the star observing time if the star is circum-polar and is the time duration where observation is possible when the Sun is -8 degrees below horizon.

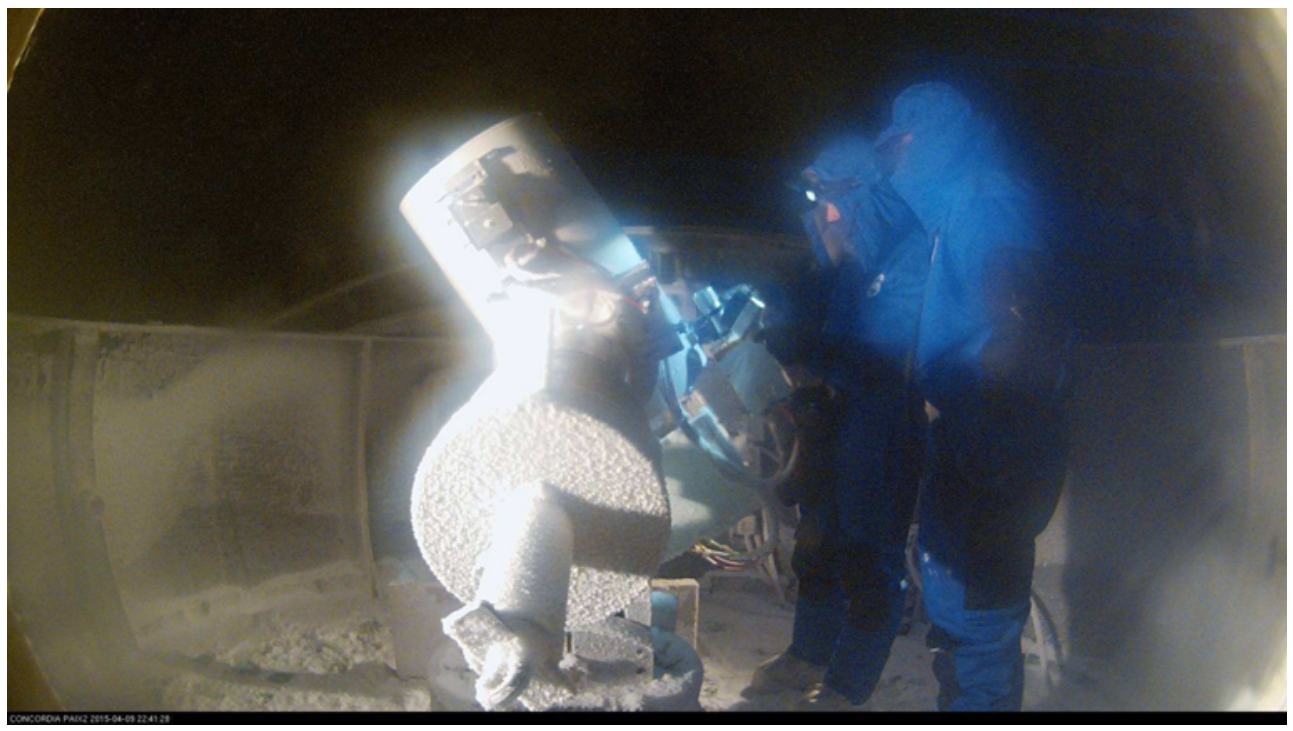

Figure 2. PAIX at Dome $\mathrm{C}$ during a quick check before starting the polar observing run 

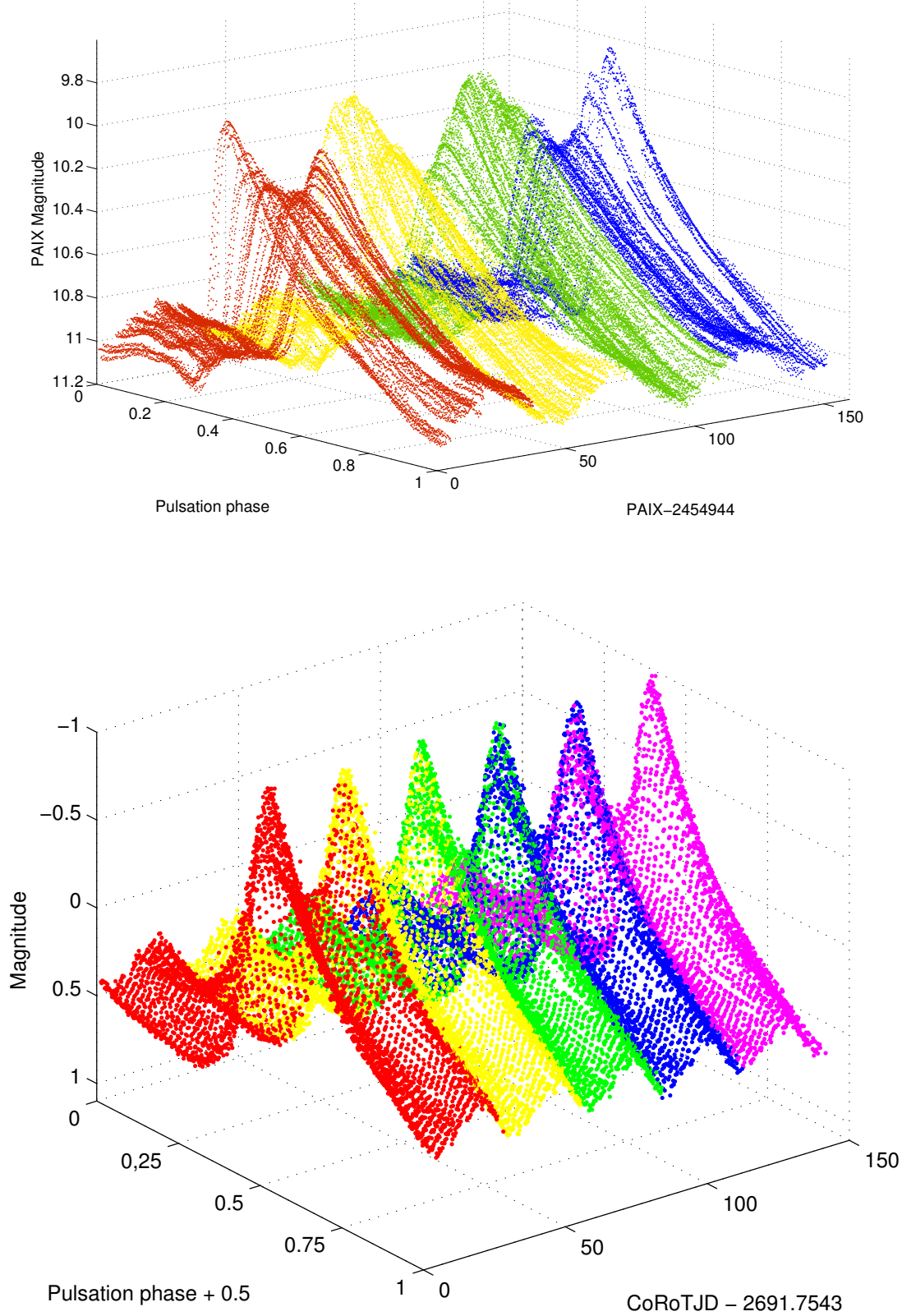

Figure 3. Top: two-dimensional PAIX light curve of the RR Lyrae star S Arae folded with the pulsation period (0.452 d) over 3 Blazhko cycles, 150 days, and showing a strong Blazhko strength. Bottom: two-dimensional CoRoT light curve of RR Lyrae star V1127 Aql folded with the pulsation period ( $0.356 \mathrm{~d})$ over five Blazhko cycles, 150 days. The variations in the light curve due to the Blazhko effect are clearly visible. 


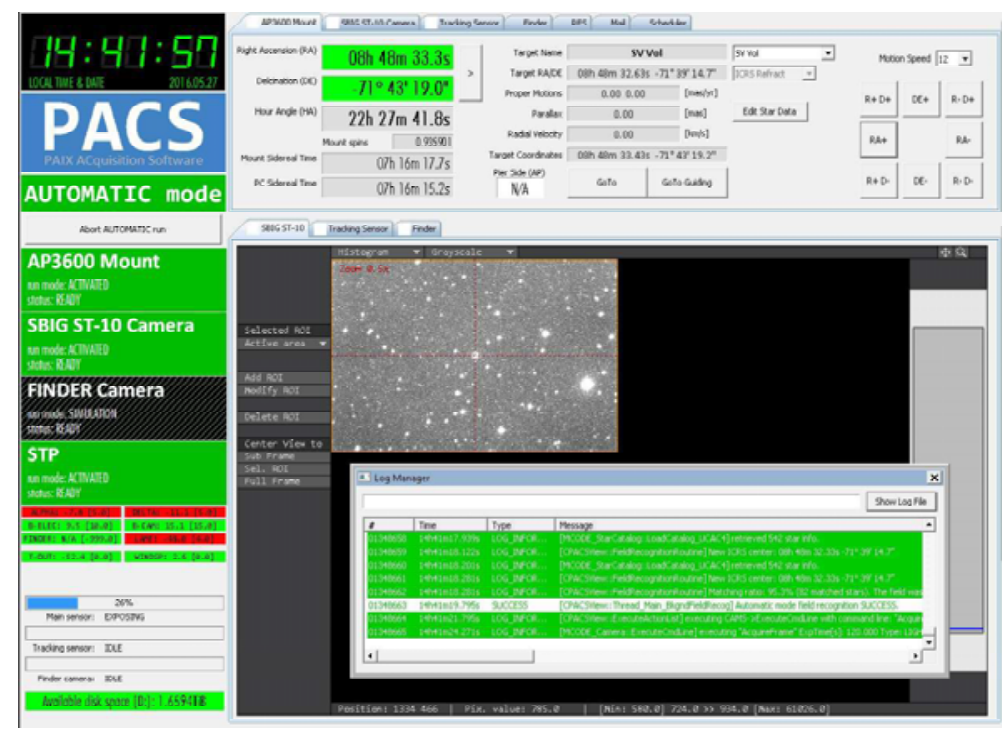

Figure 4. PACS Control Window. All PAIX components and devices are visible and remotely ordered.

instrument. The PAIX Pipeline package - PP - provides work flows for processing PAIX observed photometric data. The PAIX pipeline is dedicated to reduce the raw images and to measure both magnitude and extinction extractions towards light-curves and Bouger's lines simultaneously ([7]). This completely makes PAIX a robotic telescope, and an automatic experiment towards an executing of the polar night observing runs without any local human intervention. Thus, this new experience is a great step forward for the stellar oscillation in Antarctica and gives, for the first time, new insights in remote polar observing runs and robotic polar instruments towards new polar technology developments.

\section{Scientific impact and results}

\subsection{Star-hunting Antarctica}

The fields of stellar evolution and pulsation, have been flourished during the last few years. The new generation of largest optical and infrared ground-based telescopes with 8-10 m class, VLT, Keck, LBT and Subaru, are providing major constraints on the pulsation and evolutionary properties, the surface abundances, the internal stellar structure and the lifetimes of stellar populations in the nearby Universe. These observational data have been thoroughly complemented with high resolution images and time-series photometric data from space telescopes, HST, Chandra, Spitzer, MOST, CoRoT and Kepler. Despite this incredible observational progress, we are facing big challenges and many old and new astrophysical issues for which we still lack a robust explanation. The new circumpolar technology PAIX opens wide eyes to the Universe in helping to deal with challenges in the stellar pulsation and evolution, specially (1) the period search, search for secondary modulations and their origin, and mysterious temporal hydrodynamical phenomena in RR Lyrae stars which are still not understood a century after their discovery, with large extension to several type of pulsating stars across the Hertzsprung-Russell diagram, and (2) the time-series multi-color and wide-field photometric observations of globular clusters provide the opportunity to investigate the cosmic distance scale and to study the evolutionary properties of stellar populations. 


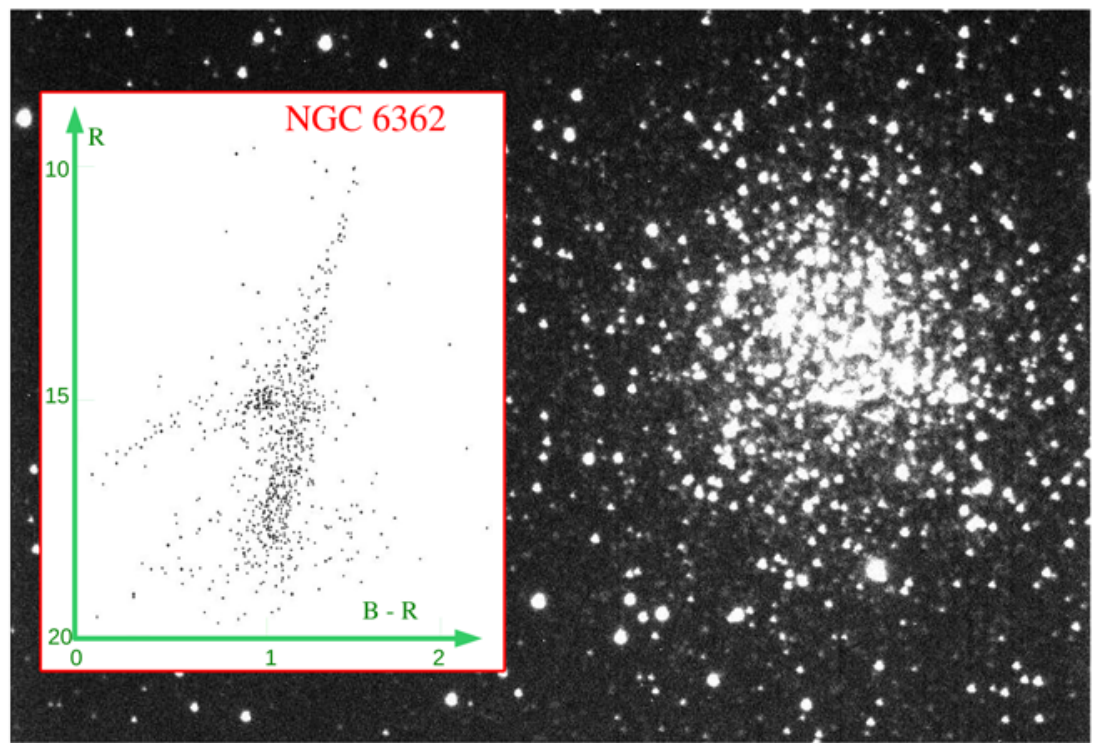

Figure 5. Globular cluster NGC 6362 and color-magnitude diagram of the observed fields based on simultaneous multi color time-series data from PAIX.

\subsection{Large photometric survey}

The PAIX polar mission is a great opportunity for monitoring stars with excellent time-sampling and unprecedented multicolor photometric precision for up to 150 days. As an important benefit, highquality RR Lyrae light curves are obtained with a quasi-uninterrupted coverage over several pulsation and Blazhko cycles. Due to the proper data sampling, our first analysis shows new results on the frequency content, the amplitude behavior, new light curve properties, physical characteristics derived from Fourier parameters, namely metal abundances, mass, temperature, surface gravities and distances. The details and strategies of the frequency analysis studies can be found in [3] and [6]. Furthermore, we described the high-temperature hydrodynamic phenomena challenges on a higher level revealing more details to constrain the theoretical models. As shown in Figure 6, we demonstrated that the atmosphere of RR Lyrae stars is crossed by a multiple shock structure intimately connected to the Blazhko modulation. Figure 7 shows a systematic change of the shock intensity with the period of the Blazhko effect. We suggested then that the Blazhko enigma in RRab stars may be explained as a dynamical coupling interplay between a multi-shock structure and an outflowing wind in a coronal structure ([6]). On the other hand, monitoring the globular clusters without the regular interruptions imposed by the earth rotation and with long-term multicolor photometric observations is an outstanding opportunity of determining accurate ages and distances of globular clusters. In the framework of an ongoing high resolution spectroscopic survey aimed at to study southern RR Lyrae stars ([5, 9]), [8] performed a spectroscopic comparison of metal-rich and metal-poor RR Lyrae stars and showed that the period distribution is different between the metal-rich Galactic field and globular cluster RR Lyrae stars. The reason of the different period distributions of these two metal-rich populations is still unknown. NGC 6362 was selected as one of the targets of our ongoing polar photometric survey aimed at identification and pulsating star study towards an understanding of the evolutionary issues of stellar population in the nearby Universe. The field of the southern hemisphere globular cluster NGC 6362 


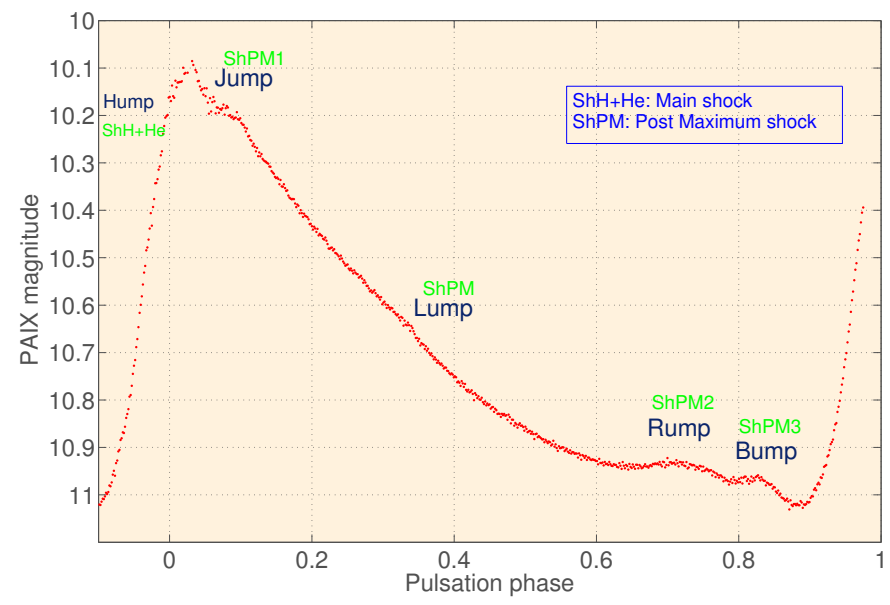

Figure 6. PAIX light curve of S Arae showing the hump, jump, lump, rump, and bump induced by shock waves.
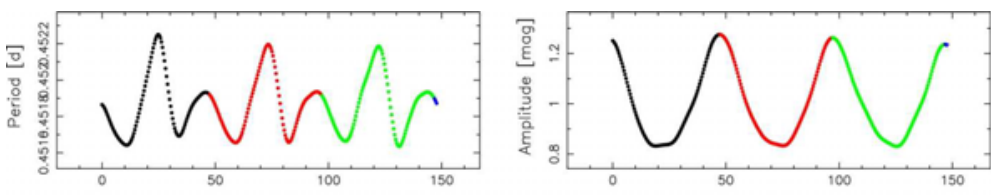

HJD -2450000

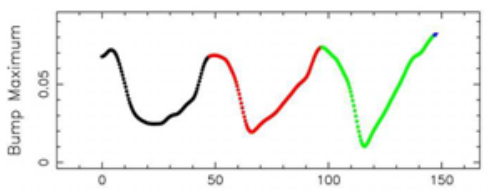

HND -2450000

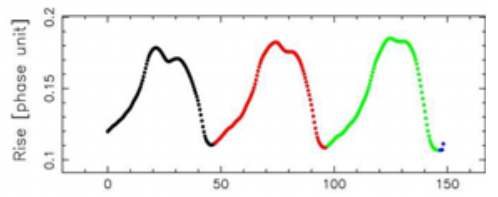

HDS -2450000

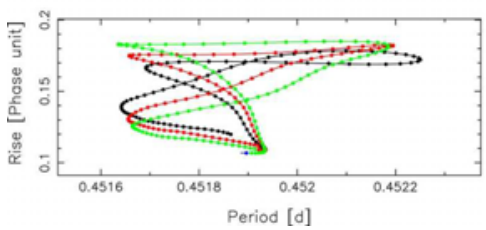

HDJ -2450000

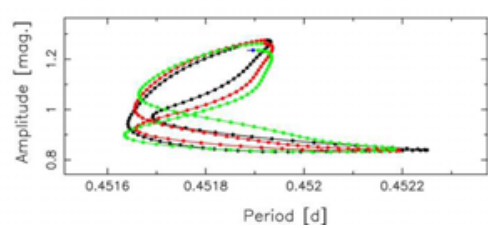

Figure 7. Pulsation period, amplitude, bump maximum, and rise time as a function of time. The rise-period diagram and the amplitude-period diagram are shown at the first (black), second (red), and third (green) Blazhko cycles of S Arae.

has been monitored over one polar night, 150 days, simultaneous uninterrupted $U B V R I$-band photometric data were collected and Fourier decomposition of the light curves is used to estimate the basic properties of cluster variables. Figure 5 presents a color-magnitude diagram $(R$ magnitudes versus $B-V$ colors) from our ongoing NGC 6362 data analysis. 


\section{Science for less money}

PAIX results demonstrate that Antarctic observations are scientifically and financially competitive with space missions. In fact, in some area, Antarctic Sciences compete with spatial missions notwithstanding development costs which are not comparable. A brief comparison between CoRoT ([3]) and PAIX ([6]) shows that PAIX scientifically challenges the CoRoT space mission (Fig. 3) and even exceeds the space mission with more advantages in multi-color time-series performances. The total PAIX cost was around $150 \mathrm{k} €$, including various costs related to the facilities in terms of Dome C base building, maintenance and polar expeditions. In comparison, the CoRoT satellite mission cost 50 $\mathrm{M} €$, without including the related price of Baïkonour Cosmodrome base, ESA and CNES agencies and Thales Aliena company. Besides the financial side, technical gaps arise between space and polar missions as evidenced by the fact that the polar missions show a large flexibility during the observing runs in choosing the targets, in changing the observation set-up and the repair of any failures that happen during the observing runs.

Acknowledgments: I warmly thank Hiromoto Shibahashi for having given this invited talk on behalf of me during my endurance in Antarctica. I would also to acknowledge Márcio Catelan and Wolfgang Gieren for the invitation to give this talk and the considerable effort required to organize the San Pedro LASPCS 2016 meeting.

\section{References}

[1] Baglin, A., Auvergne, M., Catala, C., Michel, E., Goupil, M. J., Samadi, R., Popielsky, B., \& COROT Team, in IAU Colloq. 185: Radial $\mathcal{F}$ Nonradial Pulsations as Probes of Stellar Physics, ed. C. Aerts, T. R. Bedding, \& J. Christensen- Dalsgaard, Astronomical Society of the Pacific Conference Series, 259, 626 (2002)

[2] Borucki, W. J., Koch, D. G., Lissauer, J., et al., in Transiting Extrapolar Planets Workshop, ed. C. Afonso, D. Weldrake, \& T. Henning, Astronomical Society of the Pacific Conference Series, 366, 309 (2007)

[3] Chadid, M., Vernin, J., Trinquet, H., \& Bono, G., A\&A, 516, L15 (2010)

[4] Chadid, M., et al., A\&A, 510, 39 (2010)

[5] Chadid, M., \& Preston, G. W., MNRAS, 434, 552 (2013)

[6] Chadid, M., Vernin, J., Preston, G., Zalian, C., Pouzenc, C., Aristidi, E., Liu, L. Y., \& Trinquet, H., AJ, 148, 88 (2014)

[7] Chadid, M., Vernin, J., Abe, L., et al., Proceedings SPIE, Volume 9908 (2016)

[8] Chadid, M., Sneden, C., \& Preston, G. W., ApJ, 835, 187 (2017)

[9] For, B.-Q., Preston, G. W., \& Sneden, C., ApJS, 194, 38 (2011)

[10] Giordano, C., Vernin, J., Chadid, M., Aristidi, E., Agabi, A., \& Trinquet, H., PASP, 124, 494 (2012)

[11] Lawrence, J. S., Ashley, M. C. B., Tokovinin, A., \& Travouillon, T., Nature, 431, 278 (2004)

[12] Matthews, J., J. of the Roy. Astron. Soc. of Canada, 92, 314 (1998)

[13] Vernin, J., Chadid, M., Aristidi, E., Agabi, A., Trinquet, H., \& van der Swaelmen, M., A\&A, 500, 1271 (2009)

[14] Vernin, J., Chadid, M., Aristidi, E., Azouit, M., Sadibekova, T., \& Trinquet, H., Highlights of Astronomy, 14, 695 (2007)

[15] Trinquet, H., Agabi, A., Vernin, J., Azouit, M., Aristidi, E., \& Fossat, E., PASP, 120, 203 (2008) 